\title{
String Theory and Non-Riemannian Geometry
}

\author{
Jeong-Hyuck Park (朴廷爀) $\oplus^{1,2, * *}$ and Shigeki Sugimoto (杉本茂樹) ${ }^{2, \dagger}$ \\ ${ }^{1}$ Department of Physics, Sogang University, 35 Baekbeom-ro, Mapo-gu, Seoul 04107, Korea \\ ${ }^{2}$ Center for Gravitational Physics, Yukawa Institute for Theoretical Physics, Kyoto University, \\ Kitashirakawa Oiwakecho, Sakyo-ku, Kyoto 606-8502, Japan
}

(Received 11 August 2020; accepted 14 October 2020; published 19 November 2020)

\begin{abstract}
The $\mathbf{O}(D, D)$ covariant generalized metric, postulated as a truly fundamental variable, can describe novel geometries where the notion of Riemannian metric ceases to exist. Here we quantize a closed string upon such backgrounds and identify flat, anomaly free, non-Riemannian string vacua in the familiar critical dimension, $D=26$ (or $D=10$ ). Remarkably, the whole Becchi-Rouet-Stora-Tyutin closed string spectrum is restricted to just one level with no tachyon, and matches the linearized equations of motion of double field theory. Taken as an internal space, our non-Riemannian vacua may open up novel avenues alternative to traditional string compactification.
\end{abstract}

DOI: 10.1103/PhysRevLett.125.211601

Motivation: Absence of tachyon kinetic term in double field theory.-Ever since the adoption of Riemannian geometry into the formulation of general relativity, the metric $g_{\mu \nu}$ has been privileged to be the fundamental variable that provides a concrete mathematical tool to address the notion of spacetime. In particular, the "flat" spacetime where the gravitational effect is negligible is simply given by a constant metric of Minkowskian signature. Needless to say, the standard model upon this background is arguably the best tested theory.

The flat Minkowskian spacetime is famously known to be unstable in bosonic string theory, as both the open and closed string spectra contain a negative mass-squared tachyon. Open string tachyon means the instability of a D-brane. Its tachyon potential has a local minimum which corresponds to a closed string vacuum without any D-brane [1-5]. Yet, for the closed string tachyon, little is known except the effective description,

$$
\begin{gathered}
\int d^{D} x \sqrt{-g} e^{-2 \phi}\left[R+4 \partial_{\mu} \phi \partial^{\mu} \phi-\frac{1}{12} H_{\lambda \mu \nu} H^{\lambda \mu \nu}\right. \\
\left.-\frac{2(D-26)}{3 \alpha^{\prime}}-\partial_{\mu} T \partial^{\mu} T+\frac{4}{\alpha^{\prime}} T^{2}+\mathcal{O}\left(T^{3}\right)\right] .
\end{gathered}
$$

In this Letter, we construct a novel bosonic closed string theory with a finite spectrum free of tachyon, by going beyond the Riemannian paradigm. Our working hypothesis, motivated by $T$ duality, is to view an $\mathbf{O}(D, D)$

Published by the American Physical Society under the terms of the Creative Commons Attribution 4.0 International license. Further distribution of this work must maintain attribution to the author(s) and the published article's title, journal citation, and DOI. Funded by SCOAP ${ }^{3}$. invariant metric, $\mathcal{J}_{M N}$, and an $\mathbf{O}(D, D)$ covariant generalized metric, $\mathcal{H}_{M N}$, as fundamental entities (instead of $g_{\mu \nu}$ ). The former is put in an off-block diagonal form, $\mathcal{J}_{M N}=\left(\begin{array}{ll}\mathbf{0} & \mathbf{1} \\ \mathbf{1} & \mathbf{0}\end{array}\right)$, and, with its inverse, lowers and raises the $\mathbf{O}(D, D)$ indices, capital Roman $M, N=1,2, \ldots, 2 D$. The latter then satisfies twofold defining properties:

$$
\mathcal{H}_{M N}=\mathcal{H}_{N M}, \quad \mathcal{H}_{M}{ }^{K} \mathcal{H}_{N}{ }^{L} \mathcal{J}_{K L}=\mathcal{J}_{M N}
$$

A famous parametrization reads [6]

$$
\mathcal{H}_{M N}=\left(\begin{array}{cc}
g^{\mu \nu} & -g^{\mu \sigma} B_{\sigma \lambda} \\
B_{\kappa \rho} g^{\rho \nu} & g_{\kappa \lambda}-B_{\kappa \rho} g^{\rho \sigma} B_{\sigma \lambda}
\end{array}\right),
$$

which contains $g_{\mu \nu}$ and a skew-symmetric $B$ field, corresponding to a well-known coset, $\mathbf{O}(D, D) /[\mathbf{O}(1, D-1) \times$ $\mathbf{O}(D-1,1)]$ for Minkowskian signature. Over the last three decades, this has been a cornerstone for the developments of $\mathbf{O}(D, D)$ symmetry manifest formulations of worldsheet string theory [7-14] and also target spacetime effective descriptions [15-20]. They go under the name doubled string or double field theory (DFT), as the spacetime coordinates are formally doubled, $x^{\mu} \rightarrow x^{M}=\left(\tilde{x}_{\mu}, x^{\nu}\right)$.

With Eq. (3), the closed string effective action (1) can be reformulated as a DFT coupled to the tachyon,

$$
\begin{aligned}
& \int e^{-2 d}\left[S_{(0)}-\frac{2(D-26)}{3 \alpha^{\prime}}-\mathcal{H}^{M N} \partial_{M} T \partial_{N} T\right. \\
& \left.+\frac{4}{\alpha^{\prime}} T^{2}+\mathcal{O}\left(T^{3}\right)\right] .
\end{aligned}
$$

Here $d$ is the $\mathbf{O}(D, D)$ singlet DFT dilaton related to the conventional dilaton through $e^{-2 d}=\sqrt{-g} e^{-2 \phi}$, and $S_{(0)}$ 
denotes the scalar curvature in DFT which can be spelled out explicitly in terms of $d, \mathcal{H}_{M N}$, and $\mathcal{J}_{M N}$ [20]:

$$
\begin{aligned}
S_{(0)}= & \frac{1}{8} \mathcal{H}^{M N} \partial_{M} \mathcal{H}_{K L} \partial_{N} \mathcal{H}^{K L}+\frac{1}{2} \mathcal{H}^{M N} \partial^{K} \mathcal{H}_{M L} \partial^{L} \mathcal{H}_{N K} \\
& -\partial_{M} \partial_{N} \mathcal{H}^{M N}+4 \partial_{M}\left(\mathcal{H}^{M N} \partial_{N} d\right)-4 \mathcal{H}^{M N} \partial_{M} d \partial_{N} d .
\end{aligned}
$$

The so-called section condition should be imposed on the doubled coordinates, $\partial_{M}=\left(\tilde{\partial}^{\mu}, \partial_{\nu}\right)$,

$$
\partial_{M} \partial^{M}=\partial_{\mu} \tilde{\partial}^{\mu}+\tilde{\partial}^{\mu} \partial_{\mu} \equiv 0,
$$

such that all quantities have $D$-dimensional halved dependence. After solving the section condition by letting $\tilde{\partial}^{\mu} \equiv 0$ and assuming the Riemannian parametrization (3), the DFT action (4) reduces to Eq. (1).

Crucially, Eq. (3) is not the most general solution to the defining relations (2). It only becomes so if the upper left $D \times D$ block, i.e., $\mathcal{H}^{\mu \nu}$, is invertible. DFT works perfectly fine with any generalized metric that satisfies Eq. (2). In particular, the $D \times D$ block can be degenerate, hence non-Riemannian by nature [21-27] (see also Refs. [28-33] for supersymmetric or exceptional examples). The most general parametrizations of a generalized metric have been classified by two nonnegative integers, $(n, \bar{n})$, which set $\operatorname{dim}\left(\operatorname{ker} \mathcal{H}^{\mu \nu}\right)=n+\bar{n}$, and render string chiral and antichiral over the $n$ and $\bar{n}$ directions, respectively [23]; see also Eq. (13) later. The Riemannian geometry of Eq. (3) is of $(0,0)$ type, and nonrelativistic or ultrarelativistic strings [34-40] belong to $(1,1)$ or other non-Riemannian types [22,23,25,32].

Postulating $\left\{\mathcal{J}_{M N}, \mathcal{H}_{M N}, d\right\}$ as the only geometric quantities, one can uniquely identify a covariant derivative, $\nabla_{M}[41,42]$, and then construct the scalar curvature, $S_{(0)}$, as well as "Einstein" tensor, $G_{M N}$, which is off-shell conserved, $\nabla_{M} G^{M N}=0$ [43]. This is all analogous to general relativity, though there seems no four-index Riemann tensor [44]. Using them, one can concisely express all the equations of motion of the DFT action (4):

$\mathcal{H}^{M N} \nabla_{M} \nabla_{N} T+\frac{4}{\alpha^{\prime}} T+\mathcal{O}\left(T^{2}\right)=0, \quad G_{M N}=T_{M N}$.

The former is the tachyonic equation of motion and the latter is the "Einstein equations" in DFT [45,46]. It unifies the equations of motion of $\mathcal{H}_{M N}$ and $d$ into a single formula, equating the Einstein tensor with a generalized stress-energy tensor. For the tachyon field it reads

$$
T_{M N}=(\mathcal{J}+\mathcal{H})_{[M}{ }^{K}(\mathcal{J}-\mathcal{H})_{N]}{ }^{L} \partial_{K} T \partial_{L} T-\frac{1}{2} \mathcal{J}_{M N} T_{(0)},
$$

where $T_{(0)}=-(1 / D) T_{M}{ }^{M}$ is the $\mathbf{O}(D, D)$ singlet trace part,

$$
T_{(0)}=\mathcal{H}^{M N} \partial_{M} T \partial_{N} T-\frac{4}{\alpha^{\prime}} T^{2}-\mathcal{O}\left(T^{3}\right)+\frac{2(D-26)}{3 \alpha^{\prime}} .
$$

In particular, the equation of motion of the DFT dilaton, or the trace of the Einstein equations, implies $S_{(0)}=T_{(0)}$. Thus, in $D=26$, if the tachyon potential admits a global minimum away from $T=0$, we have $T_{(0)}<0$ and hence the background cannot be flat, $S_{(0)}<0$.

While we refer the interested readers to Sec. II of Ref. [45] for a detailed review of the above formalism, for now what suffices us is that the Einstein curvature, $G_{M N}$, vanishes for constant $\mathcal{H}_{M N}$ and $d$. Any flat background with vanishing tachyon, $T=0$, solves all the equations of motion (6) in the critical dimension, $D=26$. Surely this statement is also valid for the Riemannian action (1). The novelty here is that the DFT action (4) allows non-Riemannian geometries. With the choice of the section by $\tilde{\partial}^{\mu} \equiv 0$, the tachyon kinetic term reads $\mathcal{H}^{\mu \nu} \partial_{\mu} T \partial_{\nu} T$ which obviously vanishes when $\mathcal{H}^{\mu \nu}=0$. The vanishing kinetic term then may eliminate the instability of the static configurations: There is no dynamics for the tachyon to roll down (at least classically at linear order). The absence of the kinetic term was also discussed for tachyon condensation in open string field theory $[47,48]$, while it is a generic feature of "pregeometrical" or "purely cubic" string field theories $[49,50]$.

The generalized metric with $\mathcal{H}^{\mu \nu}=0$ is in a way "maximally" non-Riemannian, invalidating any notion of Riemannian metric, not to mention its signature. It corresponds to the $(n, \bar{n})$ type with $n+\bar{n}=D$, and assumes the most general form [23],

$\mathcal{H}_{M N}=\left(\begin{array}{cc}0 & Y_{i}^{\mu} X_{\lambda}^{i}-\bar{Y}_{\bar{l}}^{\mu} \bar{X}_{\lambda}^{\bar{\nu}} \\ X_{\kappa}^{i} Y_{i}^{\nu}-\bar{X}_{\kappa}^{\bar{\imath}} \bar{Y}_{\bar{l}}^{\nu} & 2 X_{(\kappa}^{i} B_{\lambda) \rho} Y_{i}^{\rho}-2 \bar{X}_{(\kappa}^{\bar{l}} B_{\lambda) \rho} \bar{Y}_{\bar{l}}^{\rho}\end{array}\right)$,

where $i=1,2, \ldots, n$ and $\bar{\imath}=1,2, \ldots, \bar{n}$. Viewing $\left(X_{\mu}^{i}, \bar{X}_{\mu}^{\bar{\imath}}\right)$ as a $D \times D$ matrix, $\left(Y_{i}^{\nu}, \bar{Y}_{\bar{l}}^{\nu}\right)$ is its inverse satisfying $X_{\mu}^{i} Y_{i}^{\nu}+\bar{X}_{\mu}^{\bar{i}} \bar{Y}_{\bar{l}}^{\nu}=\delta_{\mu}{ }^{\nu}$. The underlying coset is $\mathbf{O}(D, D) /[\mathbf{O}(n, n) \times \mathbf{O}(\bar{n}, \bar{n})][25]$ whose dimension $4 n \bar{n}$ matches the number of infinitesimal fluctuation modes, i.e., moduli, around the generalized metric (7) [26]. The types of $(D, 0)$ or $(0, D)$ are worthy of note. They are uniquely given by $\mathcal{H}_{M N}= \pm \mathcal{J}_{M N}$, and correspond to the most symmetric vacua of DFT with no moduli [24]. Intriguingly then, Riemannian spacetime may arise in DFT after the spontaneous symmetry breaking of $\mathbf{O}(D, D)$, which identifies $g_{\mu \nu}$ and $B_{\mu \nu}$ as the massless Nambu-Goldstone bosons [25] (cf. Refs. [51,52]).

In the remainder of this Letter, we investigate the quantum consistency of the non-Riemannian geometries (7) as for novel string vacua. Through Becchi-Rouet-StoraTyutin (BRST) quantization of the string, we show that the type of $(n, \bar{n})=(13,13)$ with $D=26$ is anomaly free. Remarkably, the string spectrum is finite with no tachyon mode, matches the coset underlying (7), and agrees with the linearized DFT equations of motion, i.e., the vacuum Einstein equations, $G_{M N}=0$. We shall conclude with remarks on extension to type II superstring and application 
as an alternative to string compactification on Riemannian manifolds.

BRST quantization of doubled-yet-gauged string.-The doubled string action we consider is [14,21],

$$
\begin{aligned}
S & =\frac{1}{4 \pi \alpha^{\prime}} \int d^{2} \sigma \mathcal{L}, \\
\mathcal{L} & =-\frac{1}{2} \sqrt{-h} h^{\alpha \beta} D_{\alpha} x^{M} D_{\beta} x^{N} \mathcal{H}_{M N}-\epsilon^{\alpha \beta} D_{\alpha} x^{M} A_{\beta M} .
\end{aligned}
$$

$D_{\alpha}$ is a covariant derivative with an auxiliary potential that satisfies a section-condition-like constraint,

$$
D_{\alpha} x^{M}:=\partial_{\alpha} x^{M}-A_{\alpha}^{M}, \quad A_{\alpha}^{M} \partial_{M}=0 .
$$

While the action is completely covariant under desired symmetries like $\mathbf{O}(D, D)$ rotations, Weyl symmetry, worldsheet as well as doubled target spacetime diffeomorphisms, it also concretely realizes the idea that the doubled coordinates in DFT are actually gauged and each gauge orbit corresponds to a single physical point [53]. The relevant "coordinate gauge symmetry" reads

$$
\delta x^{M}=\Delta^{M}, \quad \delta A_{\alpha}^{M}=\partial_{\alpha} \Delta^{M}, \quad \Delta^{M} \partial_{M}=0,
$$

which leaves $D_{\alpha} x^{L}, \mathcal{H}_{M N}$ invariant $\left(\Delta^{L} \partial_{L} \mathcal{H}_{M N}=0\right)$, and enables us to identify the first term in the Lagrangian (8) as a "proper area" in doubled geometry [54].

With the choice of the section, $\left(\tilde{\partial}^{\mu}, \partial_{\nu}\right) \equiv\left(0, \partial_{\nu}\right)$, which we henceforth assume throughout, the constraints on the gauge potential (9) and parameter (10) are solved by $A_{\alpha}^{M} \equiv\left(A_{\alpha \mu}, 0\right)$ and $\Delta^{M} \equiv\left(\Delta_{\mu}, 0\right)$. Clearly then, it is the tilde coordinates $\tilde{x}_{\mu}$ that are to be gauged: $D_{\alpha} x^{M}=\left(\partial_{\alpha} \tilde{x}_{\mu}-A_{\alpha \mu}, \partial_{\alpha} x^{\nu}\right)$.

Upon the Riemannian background (3), the potential $A_{\alpha \mu}$ appears quadratically in the action, $\left(1 / 4 \pi \alpha^{\prime}\right) \mathcal{L}=$ $\left(1 / 2 \pi \alpha^{\prime}\right) \mathcal{L}^{\prime}$,

$$
\begin{aligned}
\mathcal{L}^{\prime}= & -\frac{1}{2} \sqrt{-h} h^{\alpha \beta} \partial_{\alpha} x^{\mu} \partial_{\beta} x^{\nu} g_{\mu \nu}+\frac{1}{2} \epsilon^{\alpha \beta} \partial_{\alpha} x^{\mu} \partial_{\beta} x^{\nu} B_{\mu \nu} \\
& +\frac{1}{2} \epsilon^{\alpha \beta} \partial_{\alpha} \tilde{x}_{\mu} \partial_{\beta} x^{\mu}-\frac{1}{4} \sqrt{-h} h^{\alpha \beta}\left(A_{\alpha \mu}-V_{\alpha \mu}\right)\left(A_{\beta \nu}-V_{\beta \nu}\right) g^{\mu \nu},
\end{aligned}
$$

where $V_{\alpha \mu}=\partial_{\alpha} \tilde{x}_{\mu}-B_{\mu \nu} \partial_{\alpha} x^{\nu}+(1 / \sqrt{-h}) \epsilon_{\alpha}{ }^{\beta} g_{\mu \nu} \partial_{\beta} x^{\nu}$, and the worldsheet indices are raised (lowered) with $h^{\alpha \beta}\left(h_{\alpha \beta}\right)$. Integrating out the auxiliary potential and further fixing the coordinate gauge symmetry by $\tilde{x}_{\mu} \equiv 0$ (cf. Ref. [55]), one recovers the familiar (Riemannian) string action.

We now focus on the maximally non-Riemannian constant background (7). For simplicity, we ignore the $B$ field and diagonalize the square matrices, $\left(X_{\mu}^{i}, \bar{X}_{\mu}^{\bar{\imath}}\right),\left(Y_{i}^{\nu}, \bar{Y}_{\bar{l}}^{\nu}\right)$, to be identity matrices. The $D$-dimensional index $\mu$ decomposes into two parts: $\mu=(i, \bar{l})$. We perform a field redefinition of the potential $A_{\alpha \mu}$ to a coordinate gauge symmetry invariant quantity, $p_{\alpha \mu}$,

$$
p_{\alpha i}:=\partial_{\alpha} \tilde{x}_{i}-A_{\alpha i}, \quad p_{\alpha \bar{l}}:=A_{\alpha \bar{l}}-\partial_{\alpha} \tilde{x}_{\bar{l}},
$$

and prepare a pair of projection matrices from [56]

$h_{ \pm}^{\alpha \beta}=h_{\mp}^{\beta \alpha}=\frac{1}{2}\left(h^{\alpha \beta} \pm \frac{\epsilon^{\alpha \beta}}{\sqrt{-h}}\right), \quad \begin{gathered}h_{ \pm \alpha}{ }^{\beta} h_{ \pm \beta^{\gamma}}{ }^{\gamma}=h_{ \pm \alpha}{ }^{\gamma}, \\ h_{ \pm \alpha}{ }^{\beta} h_{\mp \beta}{ }^{\gamma}=0 .\end{gathered}$

The string Lagrangian (8) now assumes the form

$\mathcal{L}_{0}=-\sqrt{-h}\left(p_{\alpha i} h_{+}^{\alpha \beta} \partial_{\beta} x^{i}+p_{\alpha \bar{l}} h_{-}^{\alpha \beta} \partial_{\beta} x^{\bar{i}}\right)+\epsilon^{\alpha \beta} \partial_{\alpha} \tilde{x}_{\mu} \partial_{\beta} x^{\mu}$.

Evidently, $p_{\alpha \mu}$ 's are Lagrange multipliers imposing the chirality and antichirality on the untilde coordinates: $h_{+}^{\alpha \beta} \partial_{\beta} x^{i} \equiv 0$ and $h_{-}^{\alpha \beta} \partial_{\beta} x^{\bar{i}} \equiv 0$ [21,23] (cf. Refs. [57-59] which are fully chiral and equipped with a Riemannian metric).

Toward the BRST quantization, it is convenient to parametrize $\sqrt{-h} h^{\alpha \beta}$, which has unit determinant, by two variables, without loss of generality,

$$
\sqrt{-h} h^{\tau \tau}=-\frac{1}{e}, \quad \sqrt{-h} h^{\tau \sigma}=\frac{\omega}{e}, \quad \sqrt{-h} h^{\sigma \sigma}=e-\frac{\omega^{2}}{e} .
$$

Under diffeomorphisms $\delta_{c} \sigma^{\alpha}=c^{\alpha}$ these two transform,

$\delta_{c} e=c^{\alpha} \partial_{\alpha} e+\left(\partial_{\tau} c^{\tau}-\partial_{\sigma} c^{\sigma}\right) e-2 \partial_{\sigma} c^{\tau} \omega e$,

$\delta_{c} \omega=c^{\alpha} \partial_{\alpha} \omega+\left(\partial_{\tau} c^{\tau}-\partial_{\sigma} c^{\sigma}\right) \omega+\partial_{\tau} c^{\sigma}-\partial_{\sigma} c^{\tau}\left(\omega^{2}+e^{2}\right)$,

which match the standard transformation of $\sqrt{-h} h^{\alpha \beta}$. We shall also use (from time to time) the worldsheet light-cone convention,

$$
\begin{aligned}
& \sigma^{ \pm}=\tau \pm \sigma, \\
& c^{ \pm}=c^{\tau} \pm c^{\sigma}, \quad \partial_{ \pm}=\frac{1}{2}\left(\partial_{\tau} \pm \partial_{\sigma}\right), \\
& =\frac{1}{2}\left(p_{\tau \mu} \pm p_{\sigma \mu}\right) .
\end{aligned}
$$

In addition to the coordinate gauge symmetry (10) and the worldsheet diffeomorphisms (14), from Eq. (12), the Lagrangian (13) admits extra gauge symmetry:

$$
\left\{\begin{array} { l } 
{ \delta p _ { \alpha i } = h _ { + \alpha } ^ { \beta } \hat { C } _ { \beta i } } \\
{ \delta p _ { \alpha \overline { l } } = h _ { - \alpha } ^ { \beta } \hat { C } _ { \beta \overline { l } } }
\end{array} \Leftrightarrow \left\{\begin{array}{l}
\delta p_{ \pm i}=(\omega-e \pm 1) C_{i} \\
\delta p_{ \pm \bar{\imath}}=(\omega+e \pm 1) C_{\bar{l}},
\end{array}\right.\right.
$$

where $\hat{C}_{\beta \mu}$ 's are arbitrary local parameters. Yet, despite their seemingly free index, i.e., " $\beta$," since $h_{ \pm \alpha}{ }^{\beta}$ 's are $2 \times 2$ projection matrices with nontrivial kernel, the extra gauge symmetry can be specified simply by the alternative parameter $C_{\mu}$ carrying no worldsheet index. 
We proceed to fix all the gauges, (10), (14), (15):

$e \equiv 1, \quad \omega \equiv 0, \quad \tilde{x}_{\mu} \equiv 0, \quad p_{-i} \equiv 0, \quad p_{+\bar{l}} \equiv 0$,

which imply, $-\sqrt{-h} h^{\alpha \beta} \equiv\left(\begin{array}{l}\mathbf{0} \\ \mathbf{1 0}\end{array}\right)$ on the light cone and the vanishing of the topological term in Eq. (13).

The full Lagrangian with Faddeev-Popov ghosts is

$$
\mathcal{L}_{\text {full }}=\mathcal{L}_{0}-i \delta_{\mathbf{B}}\left(\ln e b_{e}+\omega b_{\omega}+\tilde{x}_{\mu} \tilde{B}^{\mu}+p_{-i} B^{i}+p_{+\bar{\imath}} B^{\bar{\imath}}\right),
$$

where $\left\{b_{e}, b_{\omega}, \tilde{B}^{\mu}, B^{\mu}\right\}$ are the antighosts for the gauge symmetries of Eqs. (14), (10), and (15). With the associated ghosts, $\left\{c^{\alpha}, \tilde{C}_{\mu}, C_{\mu}\right\}$, and auxiliary Nakanishi-Lautrup fields, $\left\{\kappa_{e}, \kappa_{\omega}, \tilde{\kappa}^{\mu}, \kappa^{\mu}\right\}$, the BRST transformations are

$$
\begin{aligned}
\delta_{\mathbf{B}} x^{\mu} & =c^{\alpha} \partial_{\alpha} x^{\mu}, \quad \delta_{\mathbf{B}} \tilde{x}_{\mu}=c^{\alpha} \partial_{\alpha} \tilde{x}_{\mu}+\tilde{C}_{\mu}, \\
\delta_{\mathbf{B}} p_{ \pm i} & =(\omega-e \pm 1) C_{i}+c^{\alpha} \partial_{\alpha} p_{ \pm i}+\partial_{ \pm} c^{+} p_{+i}+\partial_{ \pm} c^{-} p_{-i}, \\
\delta_{\mathbf{B}} p_{ \pm \bar{l}} & =(\omega+e \pm 1) C_{\bar{l}}+c^{\alpha} \partial_{\alpha} p_{ \pm \bar{l}}+\partial_{ \pm} c^{+} p_{+\bar{l}}+\partial_{ \pm} c^{-} p_{-\bar{l}}, \\
\delta_{\mathbf{B}} c^{\alpha} & =c^{\beta} \partial_{\beta} c^{\alpha}, \quad \delta_{\mathbf{B}} \tilde{C}_{\mu}=c^{\alpha} \partial_{\alpha} \tilde{C}_{\mu}, \\
\delta_{\mathbf{B}} C_{i} & =c^{\alpha} \partial_{\alpha} C_{i}+(\omega-e) \partial_{\sigma} c^{\tau} C_{i}+\partial_{\sigma} c^{\sigma} C_{i}, \\
\delta_{\mathbf{B}} C_{\bar{l}} & =c^{\alpha} \partial_{\alpha} C_{\bar{l}}+(\omega+e) \partial_{\sigma} c^{\tau} C_{\bar{l}}+\partial_{\sigma} c^{\sigma} C_{\bar{l}}, \\
\delta_{\mathbf{B}} b_{e} & =i \kappa_{e}, \quad \delta_{\mathbf{B}} b_{\omega}=i \kappa_{\omega}, \quad \delta_{\mathbf{B}} \tilde{B}^{\mu}=i \tilde{\kappa}^{\mu}, \quad \delta_{\mathbf{B}} B^{\mu}=i \kappa^{\mu}, \\
\delta_{\mathbf{B}} \kappa_{e} & =\delta_{\mathbf{B}} \kappa_{\omega}=\delta_{\mathbf{B}} \tilde{\kappa}^{\mu}=\delta_{\mathbf{B}} \kappa^{\mu}=0,
\end{aligned}
$$

while $\delta_{\mathbf{B}} e=\delta_{c} e$ and $\delta_{\mathbf{B}} \omega=\delta_{c} \omega$ are already given in Eq. (14), promoting the diffeomorphism parameters $c^{\alpha}$ as ghosts. The transformations are off-shell nilpotent, $\delta_{\mathbf{B}}^{2}=0$.

From the variational principle, setting $b_{e} \equiv b_{++}+b_{--}$, $b_{\omega} \equiv b_{++}-b_{--}$, and similarly for $\kappa_{e}, \kappa_{\omega}$, we acquire

$$
\begin{gathered}
p_{+i} \partial_{+} x^{i}+2 i b_{++} \partial_{+} c^{+}+i\left(\partial_{+} b_{++}\right) c^{+}=\kappa_{++}, \\
p_{-\bar{\imath}} \partial_{-} x^{\bar{\imath}}+2 i b_{--} \partial_{-} c^{-}+i\left(\partial_{-} b_{--}\right) c^{-}=\kappa_{--}, \\
p_{-i}=p_{+\bar{\imath}}=\tilde{\kappa}^{\mu}=\kappa^{\mu}=\tilde{B}^{\mu}=\tilde{C}_{\mu}=B^{\mu}=C_{\mu}=0,
\end{gathered}
$$

and the left-moving (right-moving) chiral (antichiral) properties,

$$
\begin{aligned}
& \partial_{-} x^{i}=0, \quad \partial_{-} p_{+i}=0, \quad \partial_{-} c^{+}=0, \quad \partial_{-} b_{++}=0, \\
& \partial_{+} x^{\bar{l}}=0, \quad \partial_{+} p_{-\bar{l}}=0, \quad \partial_{+} c^{-}=0, \quad \partial_{+} b_{--}=0,
\end{aligned}
$$

which can be also derived from the reduced Lagrangian,

$\mathcal{L}_{\text {red }}=2\left(p_{+i} \partial_{-} x^{i}+p_{-\bar{l}} \partial_{+} x^{\bar{i}}+i b_{++} \partial_{-} c^{+}+i b_{--} \partial_{+} c^{-}\right)$.

Naturally, $\left\{p_{+i}, p_{-\bar{l}}\right\}$ are identified as the conjugate momenta of $\left\{x^{i}, x^{\bar{\imath}}\right\}$, forming $D$ pairs of the " $\beta \gamma$ " system $[56,60]$ with the conformal weights 1 and 0 , for $\beta_{i} \equiv p_{+i}, \bar{\beta}_{\bar{\imath}} \equiv p_{-\bar{l}}, \quad$ and $\quad \gamma^{j} \equiv x^{j}, \bar{\gamma}^{\bar{\jmath}} \equiv x^{\bar{j}}, \quad$ respectively. Each pair contributes to a central charge by two.

The BRST charge decomposes $Q_{\mathbf{B}}=Q_{\mathbf{L}}+Q_{\mathbf{R}}$ with

$$
\begin{aligned}
Q_{\mathbf{L}} & =\oint d \sigma \beta_{i} \partial_{+} \gamma^{i} c^{+}+i\left(b_{++} \partial_{+} c^{+}\right) c^{+} \\
& =: \sum_{m, n=-\infty}^{\infty} n\left(-i \beta_{m i} \gamma_{n}^{i}+b_{m} c_{n}\right) c_{-m-n}:-a c_{0},
\end{aligned}
$$

and mirroring expression for $Q_{\mathbf{R}}$. The quantization is given by $\left[\gamma_{m}^{i}, \beta_{n j}\right]=i \delta_{j}^{i} \delta_{m+n}$ and $\left\{b_{m}, c_{n}\right\}=\delta_{m+n}$, which generate the normal ordering constant $a$. The BRST charges, $Q_{\mathbf{L}}, Q_{\mathbf{R}}$, are nilpotent, if and only if $n=\bar{n}=13$, implying the usual critical dimension, $D=26$, since the central charges are $\mathbf{c}_{\mathbf{L}}=2 n-26$ and $\mathbf{c}_{\mathbf{R}}=2 \bar{n}-26$, both of which should vanish.

Physical states are annihilated by $Q_{\mathrm{L}}$ and the antighost zero mode $b_{0}$ (mirrored by the right-moving sector). Their anticommutator is

$$
L_{0}=\left\{b_{0}, Q_{\mathbf{B}}\right\}=N_{\beta}+N_{\gamma}+N_{b}+N_{c}-a,
$$

where

$$
\begin{aligned}
& N_{\beta}=\sum_{p=1}^{\infty}-i p \beta_{-p i} \gamma_{p}^{i}, \quad N_{\gamma}=\sum_{p=1}^{\infty} i p \gamma_{-p}^{i} \beta_{p i}, \\
& N_{b}=\sum_{p=1}^{\infty} p b_{-p} c_{p}, \quad N_{c}=\sum_{p=1}^{\infty} p c_{-p} b_{p},
\end{aligned}
$$

are the level-counting operators for each creation operator with $p \geq 1$. These are all positive semidefinite. Hence, the vanishing of $L_{0}$ (21) on physical states means a drastic truncation of the entire string spectrum to just one level. Computing $\left\langle 0\left|\left[L_{1}, L_{-1}\right]\right| 0\right\rangle=-2$ with $L_{n}=\left\{Q_{\mathbf{L}}, b_{n}\right\}$, we identify the level to be unity, $a=1$. Then, from $\beta_{0 i}|k\rangle=k_{i}|k\rangle, Q_{\mathbf{L}} c_{-1}|k\rangle=Q_{\mathbf{L}} \gamma_{-1}^{i}|k\rangle=0$, and

$Q_{\mathbf{L}} \beta_{-1 i}|k\rangle=k_{i} c_{-1}|k\rangle, \quad Q_{\mathbf{L}} b_{-1}|k\rangle=\left(i k_{i} \gamma_{-1}^{i}+2 c_{-1} b_{0}\right)|k\rangle$,

the physical states consist of four sectors, (with $|k \downarrow\rangle$ satisfying $\left.b_{0}|k \downarrow\rangle=0[56,60]\right)$,

$\delta \mathcal{H}_{i \bar{\imath}} \gamma_{-1}^{i}\left|k_{j} \downarrow\right\rangle \otimes \bar{\gamma}_{-1}^{\bar{\imath}}\left|k_{\bar{\jmath}} \downarrow\right\rangle, \quad \delta \mathcal{H}_{i}^{\bar{i}} \gamma_{-1}^{i}\left|k_{j} \downarrow\right\rangle \otimes \bar{\beta}_{-1 \bar{l}}\left|k_{\bar{j}} \downarrow\right\rangle$, $\delta \mathcal{H}_{\bar{\imath}}^{i} \beta_{-1 i}\left|k_{j} \downarrow\right\rangle \otimes \bar{\gamma}_{-1}^{\bar{\imath}}\left|k_{\bar{J}} \downarrow\right\rangle, \quad \delta \mathcal{H}^{\bar{u}} \beta_{-1 i}\left|k_{j} \downarrow\right\rangle \otimes \bar{\beta}_{-1 \bar{\imath}}\left|k_{\bar{J}} \downarrow\right\rangle$,

which should satisfy on-shell relations for $Q_{\mathbf{B}}$ closedness,

$k_{\bar{\imath}} \delta \mathcal{H}_{i}^{\bar{l}}=0, \quad k_{i} \delta \mathcal{H}^{i}{ }_{\bar{\imath}}=0, \quad k_{i} \delta \mathcal{H}^{\bar{\imath}}=0, \quad k_{\bar{\imath}} \delta \mathcal{H}^{i \bar{\imath}}=0$,

and equivalence relations as for gauge symmetries, 


$$
\begin{gathered}
\delta \mathcal{H}_{i \bar{l}} \sim \delta \mathcal{H}_{i \bar{l}}+k_{i} \lambda_{\bar{l}}-k_{\bar{l}} \lambda_{i}, \\
\delta \mathcal{H}_{i}^{\bar{l}} \sim \delta \mathcal{H}_{i}^{\bar{l}}+k_{i} \xi^{\bar{l}}, \quad \delta \mathcal{H}^{i}{ }_{\bar{l}} \sim \delta \mathcal{H}^{i}{ }_{\bar{l}}-k_{\bar{l}} \xi^{i},
\end{gathered}
$$

where $\xi^{i}, \xi^{\bar{l}}$ need to be divergenceless, $k_{i} \xi^{i}=k_{\bar{\imath}} \xi^{\bar{l}}=0$.

We have a good reason to denote the physical states by the same symbol as the generalized metric: the $4 n \bar{n}$ of $\left\{\delta \mathcal{H}_{\bar{l}}, \delta \mathcal{H}_{i}^{\bar{l}}, \delta \mathcal{H}^{i}{ }_{\bar{i}}, \delta \mathcal{H}^{i \bar{u}}\right\}$ are literally the moduli of the generalized metric (7) that we have been dealing with, in the diagonal form where the only nontrivial components are $\mathcal{H}_{i}{ }^{j}=\mathcal{H}^{j}{ }_{i}=\delta_{i}{ }^{j}$ and $\mathcal{H}_{\bar{l}}{ }^{\bar{j}}=\mathcal{H}^{\bar{j}}{ }_{\bar{l}}=-\delta_{\bar{l}}{ }^{\bar{j}}$ [26]. On-shell, they meet the linearized DFT equations of motion [22] (see also Ref. [61]):

$$
\begin{aligned}
& \partial_{i} \partial_{j} \delta \mathcal{H}^{\bar{j}}=0, \quad \partial_{\bar{l}} \partial_{\bar{\jmath}} \delta \mathcal{H}^{i \bar{\jmath}}=0, \quad \partial_{i} \partial_{\bar{l}} \delta \mathcal{H}^{i \bar{\imath}}=0, \\
& \partial_{i} \partial_{j} \delta \mathcal{H}^{j}{ }_{\bar{\imath}}-\partial_{\bar{\imath}} \partial_{\bar{\jmath}} \delta \mathcal{H}_{i}^{\bar{j}}+4 \partial_{i} \partial_{\bar{\imath}} \delta d=0,
\end{aligned}
$$

which enjoy local symmetries inherited from the general covariance of DFT (generalized Lie derivative, $\hat{\mathcal{L}}_{\xi} \mathcal{H}_{M N}$ ),

$$
\begin{aligned}
& \delta\left(\delta \mathcal{H}_{\bar{\imath}}^{i}\right)=\partial_{\bar{\imath}} \xi^{i}, \quad \delta\left(\delta \mathcal{H}_{i}^{\bar{l}}\right)=-\partial_{i} \xi^{\overline{\bar{l}}}, \\
& \delta\left(\delta \mathcal{H}_{i \bar{l}}\right)=\partial_{\bar{\imath}} \lambda_{i}-\partial_{i} \lambda_{\bar{\imath}}, \quad \delta(\delta d)=-\frac{1}{4}\left(\partial_{i} \xi^{i}+\partial_{\bar{\imath}} \xi^{\bar{l}}\right) .
\end{aligned}
$$

We may choose a gauge, $\delta d=0$. Remarkably then, Eqs. (22), (23) imply Eqs. (24), (25). Further, restricted to normalizable solutions, the converse appears also true. The first and second in Eq. (24) give $\partial_{j} \delta \mathcal{H}^{j \bar{\imath}}=0, \partial_{\bar{j}} \delta \mathcal{H}^{i \bar{j}}=0$, which are generically solved by $\delta \mathcal{H}^{i \bar{l}}=\epsilon^{i j k l \cdots} \epsilon^{\bar{j} \bar{j} \bar{k}} \cdots \partial_{j} \partial_{\bar{J}} \Phi_{k l \cdots \bar{k} \bar{l} \ldots}$, and hence the third holds. The last implies $\partial_{i} \partial_{j} \delta \mathcal{H}^{j}{ }_{\bar{\imath}}=\partial_{\bar{\imath}} \partial_{\bar{\jmath}} \delta \mathcal{H}_{i}{ }^{\bar{j}}=\partial_{i} \partial_{\bar{\imath}} \varphi$ for some $\varphi$. Again for normalizable solutions, we get $\partial_{j} \delta \mathcal{H}^{j}{ }_{\bar{l}}=\partial_{\bar{l}} \varphi$ and $\partial_{\bar{j}} \delta \mathcal{H}_{i}{ }^{\bar{j}}=\partial_{i} \varphi$, which can be gauged away using the remaining Eq. (25).

As the spectrum is finite, DFT itself is to be identified as string field theory around the non-Riemannian vacua. Evident from the position of the indices, it is $\delta \mathcal{H}^{i \bar{u}}$ that may condensate and reduce the "non-Riemannianity," to build up Riemannian spacetime [26].

Comments.-Our BRST charge formula (20) can be easily extended to a generic $(n, \bar{n})$ non-Riemannian background, to include $n$ pairs of chiral $\beta \gamma, \bar{n}$ pairs of antichiral $\bar{\beta} \bar{\gamma}$, and ordinary (left-right combined) $D-n-\bar{n}$ number of $x^{\mu}$. The central charges are $\mathbf{c}_{\mathbf{L} / \mathbf{R}}=D \pm(n-\bar{n})-26$, and thus necessarily $n=\bar{n}$ and $D=26$. Nonrelativistic string theories [34-37] are examples of $(n, \bar{n})=$ $(1,1)$ [62].

The necessity of putting $n=\bar{n}$ was also noted in Ref. [23] as a condition to embed non-Riemannian geometries into type II doubled superstring [29,32] or supersymmetric DFTs [63-65], the constructions of which rely on genuine $\mathbf{O}(D, D)$ covariant vielbeins rather than the Riemannian zehnbein, $e_{\mu}{ }^{a}$ [66]. The central charges should be $\mathbf{c}_{\mathbf{L} / \mathbf{R}}=D \pm(n-\bar{n})-10$, indicating that $n=\bar{n}$ nonRiemannian geometries are consistent superstring vacua in
$D=10$, which enlarges the string theory landscape far beyond the Riemannian paradigm.

Chiral string means $x^{i}(\tau, \sigma)=x^{i}(0, \tau+\sigma)$ : it is fixed in space and thus hardly interacts with one another. This classical intuition may suggest to us to explore nonRiemannian backgrounds (either flat or curved) as candidates for an internal space, alternative to string compactification traditionally on "small" Riemannian manifolds. At a glance, in the presence of external four-dimensional Minkowskian spacetime, the truncation of the string spectrum to just one level may be no longer the case as $L_{0}$ will include external $p_{\mu} p_{\nu} \eta^{\mu \nu}$, which is not positive definite. Nevertheless, since $\left[x^{i}\left(\tau_{1}, \sigma_{1}\right), x^{j}\left(\tau_{2}, \sigma_{2}\right)\right]=0$, the correlation functions of bosonic string tachyon vertex operators will have trivial dependency along all nonRiemannian directions: with $k \cdot x=k_{\mu} x^{\mu}+k_{j} x^{j}+k_{\bar{\jmath}} x^{\bar{j}}$,

$$
\left\langle\prod_{a=1}^{N}: e^{i k_{a} \cdot x\left(\tau_{a}, \sigma_{a}\right)}:\right\rangle=\delta^{D}\left(\sum_{b=1}^{N} k_{b}\right) F\left(k_{a \mu}, \tau_{a}, \sigma_{a}\right),
$$

where $F\left(k_{a \mu}, \tau_{a}, \sigma_{a}\right)$ is independent of $k_{a j}, k_{a \bar{\jmath}}$ 's. This indicates delta-function interactions on the internal $x$ space after Fourier transformation, and thus is consistent with the classical intuition of the chiral string.

We are deeply grateful to Taichiro Kugo for encouragement. J. H. P. also thanks Chris Blair and Gerben Oling for discussion. The work of J. H. P. was supported by Basic Science Research Program by the National Research Foundation of Korea (NRF) through the Grants No. NRF-2016R1D1A1B01015196 and No. NRF2020R1A6A1A03047877. The work of S. S. was supported by JSPS KAKENHI [Grant-in-Aid for Scientific Research (B)] Grant No. JP19H01897.

park@sogang.ac.kr *sugimoto@yukawa.kyoto-u.ac.jp

On sabbatical leave from Sogang University, 35 Baekbeom-ro, Mapo-gu, Seoul 04107, Korea.

[1] A. Sen, Descent relations among bosonic D-branes, Int. J. Mod. Phys. A 14, 4061 (1999).

[2] A. Sen and B. Zwiebach, Tachyon condensation in string field theory, J. High Energy Phys. 03 (2000) 002.

[3] A. A. Gerasimov and S. L. Shatashvili, On exact tachyon potential in open string field theory, J. High Energy Phys. 10 (2000) 034.

[4] D. Kutasov, M. Marino, and G. W. Moore, Remarks on tachyon condensation in superstring field theory, arXiv:hep-th/0010108.

[5] M. Schnabl, Analytic solution for tachyon condensation in open string field theory, Adv. Theor. Math. Phys. 10, 433 (2006).

[6] A. Giveon, E. Rabinovici, and G. Veneziano, Duality in string background space, Nucl. Phys. B322, 167 (1989).

[7] M. Duff, Duality rotations in string theory, Nucl. Phys. B335, 610 (1990). 
[8] A. A. Tseytlin, Duality symmetric formulation of string world sheet dynamics, Phys. Lett. B 242, 163 (1990).

[9] A. A. Tseytlin, Duality symmetric closed string theory and interacting chiral scalars, Nucl. Phys. B350, 395 (1991).

[10] M. Rocek and E.P. Verlinde, Duality, quotients, and currents, Nucl. Phys. B373, 630 (1992).

[11] A. Giveon and M. Rocek, Generalized duality in curved string backgrounds, Nucl. Phys. B380, 128 (1992).

[12] C. M. Hull, A geometry for non-geometric string backgrounds, J. High Energy Phys. 10 (2005) 065.

[13] C. M. Hull, Global aspects of T-duality, gauged sigma models and T-folds, J. High Energy Phys. 10 (2007) 057.

[14] C. M. Hull, Doubled geometry and T-folds, J. High Energy Phys. 07 (2007) 080.

[15] W. Siegel, Two Vierbein formalism for string inspired axionic gravity, Phys. Rev. D 47, 5453 (1993).

[16] W. Siegel, Superspace duality in low-energy superstrings, Phys. Rev. D 48, 2826 (1993).

[17] C. Hull and B. Zwiebach, Double field theory, J. High Energy Phys. 09 (2009) 099.

[18] C. Hull and B. Zwiebach, The gauge algebra of double field theory and Courant brackets, J. High Energy Phys. 09 (2009) 090.

[19] O. Hohm, C. Hull, and B. Zwiebach, Background independent action for double field theory, J. High Energy Phys. 07 (2010) 016.

[20] O. Hohm, C. Hull, and B. Zwiebach, Generalized metric formulation of double field theory, J. High Energy Phys. 08 (2010) 008.

[21] K. Lee and J.H. Park, Covariant action for a string in doubled yet gauged spacetime, Nucl. Phys. B880, 134 (2014).

[22] S. M. Ko, C. Melby-Thompson, R. Meyer, and J. H. Park, Dynamics of perturbations in double field theory \& nonrelativistic string theory, J. High Energy Phys. 10 (2015) 144.

[23] K. Morand and J. H. Park, Classification of non-Riemannian doubled-yet-gauged spacetime, Eur. Phys. J. C 77, 685 (2017).

[24] K. Cho, K. Morand, and J. H. Park, Kaluza-Klein reduction on a maximally non-Riemannian space is moduli-free, Phys. Lett. B 793, 65 (2019).

[25] D. S. Berman, C. D. A. Blair, and R. Otsuki, Non-Riemannian geometry of M-theory, J. High Energy Phys. 07 (2019) 175.

[26] K. Cho and J. H. Park, Remarks on the non-Riemannian sector in double field theory, Eur. Phys. J. C 80, 101 (2020).

[27] C.D. A. Blair, Non-relativistic duality and $T \bar{T}$ deformations, J. High Energy Phys. 07 (2020) 069.

[28] E. Malek, Timelike U-dualities in generalised geometry, J. High Energy Phys. 11 (2013) 185.

[29] J. H. Park, Green-Schwarz superstring on doubled-yetgauged spacetime, J. High Energy Phys. 11 (2016) 005.

[30] J. J. Fernandez-Melgarejo, J. i. Sakamoto, Y. Sakatani, and K. Yoshida, T-folds from Yang-Baxter deformations, J. High Energy Phys. 12 (2017) 108.

[31] Y. Sakatani, Type II DFT solutions from Poisson-Lie T-duality/plurality, Prog. Theor. Exp. Phys. 2019, 073 B04 (2019).

[32] C. D. A. Blair, A worldsheet supersymmetric NewtonCartan string, J. High Energy Phys. 10 (2019) 266.
[33] D. S. Berman and C. D. A. Blair, The geometry, branes and applications of exceptional field theory, arXiv:2006.09777.

[34] J. Gomis and H. Ooguri, Nonrelativistic closed string theory, J. Math. Phys. (N.Y.) 42, 3127 (2001).

[35] U.H. Danielsson, A. Guijosa, and M. Kruczenski, IIA/B, wound and wrapped, J. High Energy Phys. 10 (2000) 020.

[36] R. Andringa, E. Bergshoeff, J. Gomis, and M. de Roo, 'Stringy' Newton-Cartan gravity, Classical Quantum Gravity 29, 235020 (2012).

[37] T. Harmark, J. Hartong, and N. A. Obers, Nonrelativistic strings and limits of the AdS/CFT correspondence, Phys. Rev. D 96, 086019 (2017).

[38] E. Bergshoeff, J. Gomis, and G. Longhi, Dynamics of Carroll particles, Classical Quantum Gravity 31, 205009 (2014).

[39] C. Duval, G. W. Gibbons, P. A. Horvathy, and P. M. Zhang, Carroll versus Newton and Galilei: Two dual non-Einsteinian concepts of time, Classical Quantum Gravity 31, 085016 (2014).

[40] X. Bekaert and K. Morand, Connections and dynamical trajectories in generalised Newton-Cartan gravity II. An ambient perspective, J. Math. Phys. (N.Y.) 59, 072503 (2018).

[41] I. Jeon, K. Lee, and J. H. Park, Differential geometry with a projection: Application to double field theory, J. High Energy Phys. 04 (2011) 014.

[42] I. Jeon, K. Lee, and J. H. Park, Stringy differential geometry, beyond Riemann, Phys. Rev. D 84, 044022 (2011).

[43] J. H. Park, S. J. Rey, W. Rim, and Y. Sakatani, $\mathbf{O}(D, D)$ covariant Noether currents and global charges in double field theory, J. High Energy Phys. 11 (2015) 131.

[44] O. Hohm and B. Zwiebach, On the Riemann tensor in double field theory, J. High Energy Phys. 05 (2012) 126.

[45] S. Angus, K. Cho, and J. H. Park, Einstein double field equations, Eur. Phys. J. C 78, 500 (2018).

[46] J.H. Park, $\mathbf{O}(D, D)$ completion of the Einstein field equations, Proc. Sci. CORFU2018 (2019) 145 [arXiv: 1904.04705].

[47] L. Rastelli, A. Sen, and B. Zwiebach, String field theory around the tachyon vacuum, Adv. Theor. Math. Phys. 5, 353 (2002).

[48] H. Hata and S. Teraguchi, Test of the absence of kinetic terms around the tachyon vacuum in cubic string field theory, J. High Energy Phys. 05 (2001) 045.

[49] H. Hata, K. Itoh, T. Kugo, H. Kunitomo, and K. Ogawa, Pregeometrical string field theory: Creation of space-time and motion, Phys. Lett. B 175, 138 (1986).

[50] G. T. Horowitz, J. D. Lykken, R. Rohm, and A. Strominger, A Purely Cubic Action for String Field Theory, Phys. Rev. Lett. 57, 283 (1986).

[51] I. Kirsch, A Higgs mechanism for gravity, Phys. Rev. D 72, 024001 (2005).

[52] S. Alexander, J. D. Barrow, and J. Magueijo, Turning on gravity with the Higgs mechanism, Classical Quantum Gravity 33, 14LT01 (2016).

[53] J. H. Park, Comments on double field theory and diffeomorphisms, J. High Energy Phys. 06 (2013) 098.

[54] J. H. Park, Stringy gravity: Solving the dark problems at 'short' distance, EPJ Web Conf. 168, 01010 (2018). 
[55] D. S. Berman and N.B. Copland, The string partition function in Hull's doubled formalism, Phys. Lett. B 649, 325 (2007).

[56] M. B. Green, J. H. Schwarz, and E. Witten, Superstring Theory. Vol. 1: Introduction (Cambridge University Press, Cambridge, England, 1987).

[57] L. Mason and D. Skinner, Ambitwistor strings and the scattering equations, J. High Energy Phys. 07 (2014) 048.

[58] W. Siegel, Amplitudes for left-handed strings, arXiv: 1512.02569

[59] K. Lee, S. J. Rey, and J. A. Rosabal, A string theory which isn't about strings, J. High Energy Phys. 11 (2017) 172.

[60] J. Polchinski, String theory. Vol. 1: An introduction to the bosonic string, https://doi.org/10.1017/CBO9780511816079.

[61] O. Hohm and D. Marques, Perturbative double field theory on general backgrounds, Phys. Rev. D 93, 025032 (2016).
[62] B. S. Kim, World sheet commuting beta gamma CFT and non-relativistic string theories, Phys. Rev. D 76, 106007 (2007).

[63] O. Hohm, S. K. Kwak, and B. Zwiebach, Unification of Type II Strings and T-Duality, Phys. Rev. Lett. 107, 171603 (2011).

[64] I. Jeon, K. Lee, and J. H. Park, Supersymmetric double field theory: Stringy reformulation of supergravity, Phys. Rev. D 85, 081501(R) (2012).

[65] I. Jeon, K. Lee, J. H. Park, and Y. Suh, Stringy unification of Type IIA and IIB supergravities under $N=2 D=10$ supersymmetric double field theory, Phys. Lett. B 723, 245 (2013).

[66] A. Coimbra, C. Strickland-Constable, and D. Waldram, Supergravity as generalised geometry I: Type II theories, J. High Energy Phys. 11 (2011) 091. 